Voix et Images

voixetimages

\title{
Déjouer l'absence pour inventer une transmission féministe
}

\section{MARIE PARENT}

Volume 43, numéro 3 (129), printemps-été 2018

URI : https://id.erudit.org/iderudit/1051092ar

DOI : https://doi.org/10.7202/1051092ar

Aller au sommaire du numéro

Éditeur(s)

Université du Québec à Montréal

ISSN

0318-9201 (imprimé)

1705-933X (numérique)

Découvrir la revue

Citer ce compte rendu

PARENT, M. (2018). Compte rendu de [Déjouer l'absence pour inventer une transmission féministe]. Voix et Images, 43(3), 139-145.

https://doi.org/10.7202/1051092ar d'utilisation que vous pouvez consulter en ligne.

https://apropos.erudit.org/fr/usagers/politique-dutilisation/ 


\author{
F É M I N IS M ES \\ Déjouer l'absence pour inventer \\ une transmission féministe \\ $+++$ \\ MARIE PARENT \\ Cégep Gérald-Godin
}

C'était la première année. Il y en aura trois mille six cents autres.

Elles feront de la p'tite tempête, forêt, femme, fée; de Grand-maman ciel, et souffle, et fée. Première et première ${ }^{1}$.

Dans Mines de rien, Isabelle Boisclair pose la question: «Comment fabriquer des intellectuelles?» Pour elle, les modèles offerts aux jeunes filles sont encore trop marqués par les qualités traditionnelles de la féminité, par «l'idée qu'il faut plaire, voire servir son prochain ${ }^{2} »$. Osons reformuler la question: comment fabriquer des féministes? Il ne s'agirait pas de concevoir le programme d'un endoctrinement, mais plutôt d'imaginer une transmission de la résistance et de l'engagement. Comment donner la permission aux filles de ne pas être - de ne pas écrire - ce que l'on attend d'elles? Quelles représentations et quels discours permettront d'ouvrir l'horizon de ce qu'elles peuvent accomplir, ressentir, penser? Ces préoccupations traversent plusieurs textes publiés dans la dernière année, que l'on pense au travail de Martine Delvaux, qui, dans Le monde est à toi ${ }^{3}$, déploie cette réflexion dans le cadre de la relation mère-fille, ou à celui de Catherine Lalonde, dont le récit dévore et digère les grands textes des «femmes fortes » de la littérature québécoise, de Josée Yvon à Hélène Monette. Comment réinvestir l'histoire des femmes et de leurs luttes? Comment la garder vivante, la porter vers les nouvelles générations? Les essais commentés ici soulèvent ces questions de différentes manières, en les transposant dans trois espaces différents: celui de la classe, celui de la scène et celui du texte.

1 Catherine Lalonde, La dévoration des fées, Montréal, Le Quartanier, coll. «Série QR», 2017, p. 128.

2 Isabelle Boisclair, Lucie Joubert et Lori Saint-Martin, Mines de rien. Chroniques insolentes, Montréal, Remue-ménage, 2015, p. 143.

3 Martine Delvaux, Le monde est à toi, Montréal, Héliotrope, coll. «K», 2017, 148 p. 


\section{LA CITÉ AU CEEUR DE LA CLASSE}

Le blogue Le bal des absentes, projet porté par Julie Boulanger et Amélie Paquet, deux écrivaines et professeures au collégial, est né du constat d'un échec des cours de littérature au cégep à inclure les femmes dans les corpus étudiés. Lancé en août 2015, il présente de courts textes, écrits en alternance par Boulanger et Paquet, qui témoignent d'expériences d'enseignement d'œuvres d'écrivaines, tant classiques que contemporaines. Le livre publié deux ans plus tard ${ }^{4}$ reprend ces entrées, rassemblées dans un nouvel ordre qui contribue à mettre en relief le point de vue développé dans les textes originaux. Ce qui est proprement fascinant dans cette lecture, c'est de suivre presque en temps réel l'élaboration d'un questionnement sur les conditions nécessaires à la circulation des idées et au dialogue dans la classe, dialogue dont dépend directement l'émancipation des étudiants et étudiantes.

Pratiquement chaque texte du Bal des absentes retrace les étapes de l'enseignement d'une œuvre en particulier, des doutes et interrogations qui ont précédé le cours aux discussions qu'a provoquées le travail du texte en groupe. Boulanger et Paquet arrivent à reconstituer le processus de construction du sens qui se réalise - ou achoppe - collectivement. Chez elles, la salle de classe devient un haut lieu du politique; nous sommes plongés au cœur de la Cité, là où observer la transformation - ou la perpétuation - de certains préjugés, là où débattre des valeurs que portent les discours. Leurs récits rendent visibles les différentes sensibilités dont sont tissés les échanges. Les auteures réussissent ainsi à saisir ce qui fait de l'enseignement une expérience profondément affective. Avec une grande honnêteté, elles décrivent leurs surprises, leurs déceptions, leurs malaises face aux réactions de la classe. Par exemple, en analysant une nouvelle de Chimananda Ngozi Adichie, Paquet se heurte à la résistance de son groupe à propos du personnage d'une mère qui néglige ses obligations familiales pour poursuivre son travail d'artiste:

[C]ette idée de la femme libre était vraiment très difficile à entendre. Pour eux, elle n'était qu'une femme coupable. Sa bisexualité ne semblait pas les déranger. C'était vraiment le fait que la mère revendique son besoin de solitude et son espace de création comme un droit inaltérable qui ne leur plaisait pas. Nous étions juste incapables de discuter, eux et moi, de cette idée de la femme libre. (101)

Ici, pour une rare fois, l'échange est bloqué. Dans la plupart des cas, toutefois, Boulanger et Paquet dépeignent des classes ouvertes et coopératives, exemplaires - un portrait qu'on sent parfois idéalisé. Il faut dire que le parti pris des auteures est de prendre le contre-pied d'un certain discours sur la paresse et l'ignorance crasses des étudiants et étudiantes, une position qui relève chez elles d'une forme d'éthique de l'enseignement.

Dans Le bal des absentes, les dimensions éthique et didactique sont intimement liées. Alors que les écrits sur l'enseignement de la littérature versent parfois dans le mysticisme - l'amour du texte se transmettrait par un genre de communion

4 Julie Boulanger et Amélie Paquet, Le bal des absentes, Montréal, La Mèche, coll. «L'ouvroir», 2017, 281 p. 
spirituelle entre un professeur charismatique et une classe subjuguée - - , Paquet et Boulanger ouvrent leur atelier et partagent leurs stratégies pédagogiques (jusqu'à souligner l'efficacité de tel texte pour enseigner telle figure de style!). Mais Le bal des absentes est loin d'être un guide pratique, et on comprend vite que les "trucs» d'enseignement occupent une importance minime par rapport à leur réflexion sur la haute exigence qu'implique la position du professeur. Quelles conditions sont nécessaires pour que l'étudiant puisse s'ouvrir au langage de l'œuvre? Boulanger et Paquet établissent un climat de confiance et de liberté en choisissant de valoriser les comportements et les caractères anticonformistes - auxquels elles s'identifient fortement -, de ne pas condamner les maladresses de la langue ou les aspérités de la pensée. Surtout, elles mettent constamment en perspective leur rapport à l'autorité: «J'essaie ainsi d'être à la fois une autorité qui ne fait pas mal et qui ne cherche pas à prendre - de l'affection ou de la reconnaissance, par exemple.» (41) Tout leur engagement vise à déconstruire la posture de docilité imposée aux étudiants, en particulier aux filles, depuis leur enfance, pour leur apprendre à porter leur parole dans l'espace public.

«Nous refusons de former les filles à se ranger du côté des hommes» (25), affirment Boulanger et Paquet d'entrée de jeu. Les lectures proposées dans Le bal des absentes, de Marie-Claire Blais à Anaïs Barbeau-Lavalette, d'Emily Dickinson à Patricia Highsmith, en passant par Marina Tsvetaeva et Christa Wolfe, travaillent à donner une légitimité à des textes écrits par des femmes tenues à l'écart de la vie littéraire de leur époque, ou bien qui mettent en scène des artistes femmes marginalisées. Les auteures montrent qu'il faut encore construire la figure de l'écrivaine, laquelle reste une abstraction aux yeux de beaucoup d'étudiants et étudiantes pour qui les modèles masculins apparaissent plus libres, plus intenses:

[J]e ne pouvais pas enseigner Baudelaire comme un rebelle pour les stimuler et [ensuite] présenter Beauvoir comme une écrivaine-philosophe sérieuse et cérébrale. [...] J'ai réfléchi à la manière de leur présenter Beauvoir pour réussir à créer une complicité entre l'autrice et eux. (63)

On entend souvent que les œuvres écrites par des femmes rejoignent moins les garçons, ou que les textes représentant des réalités touchant majoritairement les femmes, comme la prostitution ou les troubles alimentaires, sont «moins littéraires». Bélanger et Paquet abattent ces idées reçues en faisant se côtoyer joyeusement les œuvres de Despentes, de Balzac et de Flaubert, celles de Jean Rhys et d'Ernest Hemingway. Ainsi tombent les hiérarchies imposées par le canon, qui perdurent dans le milieu collégial davantage que dans le milieu universitaire aujourd'hui. Leur livre est un veritable antidote contre l'ascendant des anthologies, dont la chronologie stricte et conservatrice se contente de faire apparaitre en transparence quelques écrivaines d'avant 1960, leur réservant le plus souvent le statut de «curiosité». Le bal des absentes redonne à ces textes vie et mouvement, et nous entraîne dans leur ronde endiablée. L'acte de lire y est représenté comme une performance collective, une fête, un événement puissant qui transforme à jamais ceux et celles qui y participent. 


\section{DE LA SCÈNE À LA RUE}

«À qui revient-il de nous apprendre que des femmes ont fait preuve de désobéissance pour faire partie de l'histoire ${ }^{5}$ ? » C'est la question que posent d'entrée de jeu Marie-Claude Garneau, Marie-Ėve Milot et Marie-Claude St-Laurent dans leur essai La Coalition de la robe, qui énonce le même constat que Le bal des absentes, soit l'incapacité de l'école québécoise à prendre en charge la transmission des textes écrits par des femmes, et de manière encore plus aiguë dans l'univers du thêâtre. Dans ce «documentaire indiscipliné», les trois auteures et comédiennes retracent leur propre parcours de féministes et se demandent comment assurer le passage de la révolte. Comment faire pour ne plus échapper le témoin du relais?

Garneau, Milot et St-Laurent font commencer leur histoire en 2005, sur les bancs de l'École de thêâtre du Cégep de Saint-Hyacinthe où elles se sont rencontrées. Ce printemps-là, 100000 étudiants des cégeps et universités du Québec sont en grève pour protester contre la transformation par le gouvernement Charest de 103 millions de dollars de bourses étudiantes en prêts. Les trois jeunes femmes font partie de la distribution des Troyennes de Jean-Paul Sartre, composée des finissants du programme d'interprétation, lorsque le groupe reçoit une lettre signée par «La Coalition de la robe», un trio de jeunes femmes anonymes. Cette lettre invite les étudiants à abandonner leur série de représentations pour rejoindre le mouvement de contestation. Garneau, Milot et St-Laurent seront marquées par cet appel à l'engagement frappé du sceau du féminisme, qui devient le point d'ancrage de leur enquête. Elles réalisent qu'elles ne connaissent ni l'histoire des luttes féministes, ni le thêâtre créé par les femmes à partir des années 1970, lequel n'a pas été intégré au répertoire des grands thêâtres institutionnels.

Les trois jeunes femmes partent à la rencontre de figures marquantes du féminisme québécois: Carole Fréchette et le Théâtre des Cuisines, Jovette Marchessault, le Théâtre Expérimental des Femmes, Léa Roback, Joséphine Marchand-Durand. Si l'essai s'en tenait à cet effort de mémoire, on y trouverait bien du découragement. La parole féministe est-elle condamnée à bégayer? Les jeunes femmes sont-elles contraintes de recommencer, à chaque génération, à exhumer un passé périodiquement réenterré dans les fosses de l'histoire littéraire? Mais la lecture de cet essai est enthousiasmante sur un autre plan. Les trois auteures réussissent à instaurer une méthode et une esthétique performatives de la transmission féministe. La clé de voûte est cette fameuse «Coalition de la robe» dont nous suivons les manifestations tout au long de l'essai. Cellule militante radicale et anonyme, cette organisation insaisissable utilise l'invisibilité des femmes à son profit, en fait un moyen d'action. Les seules traces matérielles de l'existence de la Coalition sont reproduites dans le livre: un article de journal, des tracts, un manifeste, des transcriptions de performances, des verbatim d'enregistrements, des extraits de carnets de création, des messages textes partagés par les trois membres. Une collection de documents qui traverse l'œuvre et troue son discours parfois un peu sage.

5 Marie-Claude Garneau, Marie-Ėve Milot et Marie-Claude St-Laurent, La Coalition de la robe. Documentaire indiscipliné, avec des illustrations de Marie Chénier, Montréal, Remue-Ménage, 2017, p. 16-17. 
Alors qu'un collectif de l'UQAM organise, pendant le printemps étudiant de 2012, une lecture publique de textes écrits uniquement par des auteurs masculins, «les filles de la Coalition de la robe [font irruption], toutes vêtues de rouge et arborant chacune un masque représentant le visage d'une auteure dramatique (malheureusement, les gens autour étaient incapables de les identifier par leurs noms)» (63). D'un même geste, l'anonymat de la manifestante est protégé et celui auquel sont confinées les écrivaines est dévoilé; par ce double jeu sur l'identité, notre ignorance de l'histoire littéraire des femmes est pointée du doigt, rendue incontournable. La couverture du livre représentant trois femmes masquées fait écho à cette dynamique. Ces trois anonymes, ce sont nos aînées, absentes des livres d'école, ce sont les auteures du livre, ce sont leurs lectrices. «Vous êtes peut-être cette Coalition» (132), suggèrent les derniers mots de l'essai. Le masque devient cette figure de l'autonomie de la transmission, mais aussi de son instabilité et de sa fragilité. Le signe d'une transmission clandestine, toujours menacée d'extinction.

«La Coalition de la robe se veut partie intégrante d'une histoire féministe, une histoire positionnée dans une actualité consciente des actions du passé et pleine d'une mémoire capable d'envisager un avenir artistique féministe et prometteur.» (55) Si la langue un peu engoncée de son manifeste évoque vaguement les résolutions d'assemblées générales, le récit de ses actions directes est beaucoup plus inspirant. Depuis sa première manifestation dans le stationnement du Cégep de Saint-Hyacinthe en 2005, la Coalition opère en marge de l'institution, déplace le masque du thêâtre vers la rue. Elle porte une impulsion qui ne demande pas la permission d'exister, qui ne se prend pas les pieds dans les conventions. Elle incarne la version la moins docile de nous-mêmes.

\section{LE TEXTE LitTÉRAIRE OU LE PASSAGE BLOQUÉ}

L'ouvrage d'Eftihia Mihelakis, La virginité en question ou Les jeunes filles sans âge ${ }^{6}$, permet de donner une perspective historique aux questions soulevées par les deux textes précédents. L'auteure se penche sur le problème de la transmission par l'entremise de la figure antique de la parthénos, qui cristallise à la fois les idées millénaires de la vierge et de la jeune fille, et celles plus contemporaines de l'adolescente et de la fille. Quelle part de la conception traditionnelle de la vierge construit encore aujourd'hui les représentations du destin de la jeune femme? En alliant histoire culturelle et analyse littéraire, cette étude propose une archéologie du passage à l'âge adulte au féminin pour mieux comprendre comment l'intégration à l'ordre social implique un scénario qui exclut nécessairement la sororité. Mihelakis dévoile les mécanismes sociaux et imaginaires qui travaillent contre la transmission du savoir et du point de vue des femmes, jusqu'à la rendre presque impossible.

Dans la Grèce antique, un état de virginité qui perdure est considéré comme un signe de maladie chez la jeune fille. Au contraire, le mariage et la procréation

6 Eftihia Mihelakis, La virginité en question ou Les jeunes filles sans âge, Montréal, Presses de l'Université de Montréal, coll. «Espace littéraire», 2017, 191 p. 
représentent la santé puisqu'ils assurent «la reproduction de corps dociles»(33), suivant la formule de Michel Foucault, qui participent à la perpétuation de l'ordre social patriarcal et hétérosexuel. La virginité constitue un statut liminaire qui doit absolument être temporaire, car sa prolongation condamne la jeune fille à la «mort civique» (60), à «une sortie du temps» (65). La vierge, peu importe son âge, est détentrice d'une "énergie sauvage» (57) qui doit être matée pour réussir son intégration sociale. Alors que le parcours initiatique pour les garçons se réalise dans la guerre, l'appartenance à une communauté de combattants et la réalisation d'exploits épiques, il se traduit chez la fille par la défloration. Cette conception téléologique du passage à l'âge adulte se transforme au XIX siècle en un regain d'intérêt pour l'étude du viol et la fétichisation de l'hymen. L'âge du mariage se voyant repoussé, la transaction que représente l'union matrimoniale n'est valable que si la valeur d'échange de la promise, dont la pureté constitue la mesure, est sauvegardée. La rupture de l'hymen devient «la cristallisation morphologique et allégorique» (91) du passage à la vie adulte comme "perte». Dans la loi, la nature du viol est donc entièrement déterminée par la protection de l'ordre social plutôt que de la fille elle-même. La fille violée se voit détournée de son destin de femme, son intégration sociale est considérée comme interrompue, ratée. Dans tous les cas, la jeune femme est condamnée à être isolée de ses semblables. Mariée, elle est renvoyée à sa fonction reproductrice. Vierge perpétuelle ou violentée, elle se voit refuser "toute forme de communauté sororale» (116), puisqu'elle est stigmatisée, objectivée et muselée par le discours de la médecine et de la loi.

Mihelakis établit la généalogie de la parthénos depuis l'Antiquité, à partir d'Antigone et d'Électre, modèles classiques de l'adolescente qui rejette la loi, refuse d'intégrer l'ordre social et reste «bloquée» dans le passage, mais aussi de figures mythologiques, telles Artémis, déesse de la chasse et de la nature sauvage, et les Érynies, chœur de «vierges abominables» qui, chez Eschyle, possède une «force de parole incontestable» (69). Toutes sont révoltées, violentes; toutes sont condamnées aux marges de la Cité. Elles « représentent ce qu'il y a de moins utile au déploiement du politique» (70). Au contraire, elles ébranlent les fondations de la loi et de l'institution. Ces figures anciennes deviennent des réservoirs de signes pour mieux comprendre les représentations étudiées dans le corpus contemporain - dont la composition, étrangement, n'est jamais présentée ni mise en perspective par Mihelakis. Le portrait dressé à travers ces lectures d'œuvres de la fin du Xx siècle et du début du XxI ${ }^{\mathrm{e}}$ siècle s'avère troublant dans la mesure où il retrace les trajectoires de jeunes femmes dont l'initiation à une sexualité hétéronormée et axée sur la reproduction constitue encore et toujours le seul moyen d'accéder au statut d'adulte et à la vie sociale. Ces œuvres présentent des parcours initiatiques avortés, où la jeune femme, le plus souvent violée, se retrouve coincée entre la mort et la vie, entre l'enfance et la vie adulte, seule dans un temps figé, hors du monde: "Les vierges inachevées deviennent des mortes restless - agitées, inquiétantes, sans répit et sans repos.» (107) Figures de l'ange chez Christine Angot et Alice Sebold, de la princesse endormie chez Elfriede Jelinek, de la vierge suicidée chez Jeffrey Eugenides, et finalement, de la bad girl, de la punk chez Virginie Despentes, toutes correspondent à ce statut liminaire. Cette dernière incarnation de la parthénos, la mauvaise fille de Despentes dans Apocalypse bébé, 
pourrait représenter un certain espoir, la désacralisation de la vierge par la colère, la laideur, l'indocilité, mais elle aussi est perdue. Rejetée par tous, elle mourra en kamikaze pour faire voler en éclats le vernis d'innocence qui lui colle à la peau. Ainsi, le rite de passage se vit une fois de plus sous le mode de la perte et du sacrifice. Le devenir-femme ne se pense pas en dehors de cet horizon de la disparition. Entre la menace de la domestication et celle de la mort, les textes étudiés ouvrent bien peu de voies possibles.

Dans cet éventail de filles liquidées, la lecture que propose Mihelakis du roman d'Alice Sebold, The Lovely Bones (2002), pointe toutefois vers un autre mode de présence pour les disparues. Le roman imagine en effet le retour sur Terre de l'esprit de Susan, une jeune femme violée et tuée, à travers Ruth, une femme bien vivante qui accepte de partager avec la morte son enveloppe corporelle pour que celle-ci puisse expérimenter les plaisirs de la chair: «Ce sacrifice suprême est une forme de courtoisie entre les filles qui éclaire le paradigme des premières relations sexuelles autrement que par la perforation de l'hymen [...].» (141) Cette figure du «corps hospitalier» qui réverbère la présence de plusieurs filles à la fois et «ressuscite» les disparues permet d'envisager autrement la question de l'intégration sociale des filles. Le rite "sacrificiel» est ici retourné comme un gant, puisque le sacrifice de l'une sert la réappropriation du corps et du désir par l'autre. Les filles vivantes deviennent des canaux de transmission par lesquels se font entendre les voix des absentes. Le devenir-femme est soudainement lié à une présence décuplée du sujet, habité par toutes celles qui sont venues avant et n'ont pu accéder à la pleine existence physique et politique.

La lecture croisée de ces trois essais permet de dépasser le constat rebattu (et déceptif) de l'invisibilisation de l'héritage des femmes pour construire une véritable politique de la transmission féministe. Se dégage de la rencontre entre ces auteures, au carrefour de l'enseignement, de la performance, de l'écriture et du militantisme, l'exigence d'occuper l'espace public, et pas seulement ses marges, en tant que chœur de filles et de femmes. À les croire, il serait possible de détourner un rite de passage millénaire marqué par l'absence à soi-même pour en faire un rite de communion. Une initiation à la vie sociale par la solidarité et le partage du savoir. Ainsi, la «formation» féministe pourrait être vécue comme une forme de ventriloquie assumée, qui consisterait à disparaître un peu soi-même pour se laisser posséder par d'autres, par la parole des mortes et des vivantes, à travers laquelle expérimenter une texture plus dense du monde. 